\title{
Efficient Hyperelastic Regularization for Registration
}

\author{
Sune Darkner ${ }^{1}$, Michael Sass Hansen ${ }^{2}$, Rasmus Larsen ${ }^{2}$, and Mads F. Hansen ${ }^{2}$ \\ 1 eScience Center, Department of Computer Science, University of Copenhagen \\ Universitetsparken 1, DK-2100 Copenhagen, Denmark \\ 2 DTU Informatics, Technical University of Denmark \\ Richard Petersens Plads, DK-2800 Lyngby, Denmark \\ darkner@diku.dk, $\{\mathrm{msh}, \mathrm{rl}, \mathrm{mfh}\} @ i m m . d t u . d k$
}

\begin{abstract}
For most image registration problems a smooth one-to-one mapping is desirable, a diffeomorphism. This can be obtained using priors such as volume preservation, certain kinds of elasticity or both. The key principle is to regularize the strain of the deformation which can be done through penalization of the eigen values of the stress tensor. We present a computational framework for regularization of image registration for isotropic hyper elasticity. We formulate an efficient and parallel scheme for computing the principal stain based for a given parameterization by decomposing the left Cauchy-Green strain tensor and deriving analytical derivatives of the principal stretches as a function of the deformation, guaranteeing a diffeomorphism in every evaluation point. Hyper elasticity allows us to handle large deformation without re-meshing. The method is general and allows for the well-known hyper elastic priors such at the Saint Vernant Kirchoff model, the Ogden material model or Riemanian elasticity. We exemplify the approach through synthetic registration and special tests as well as registration of different modalities; 2D cardiac MRI and 3D surfaces of the human ear. The artificial examples illustrate the degree of deformation the formulation can handle numerically. Numerically the computational complexity is no more than 1.45 times the computational complexity of Sum of Squared Differences.
\end{abstract}

\section{Introduction}

Registration has been the subject of intense research as it forms the basis for most quantitative methods for analyzing and tracking morphological changes. It is well known that image registration is an ill-posed problem and to obtain a meaningful solution the problem has to regularized. Simple regularizers include re-sampling and re-gridding or diffusion and linear elasticity which penalize deformation directly upon the elements of the displacement gradient. It is desirable to use a proper rotation-invariant measure such as the strain tensor. Frequently used regularization approaches are volume preservation, parameter constraints through subspace projection, or more advanced methods such as the methods we describe based on strains e.g. Riemanian elasticity. These more advanced regularizers are based on physical models such as viscosity or elasticity. The elasticity model is

A. Heyden and F. Kahl (Eds.): SCIA 2011, LNCS 6688, pp. 295-305, 2011.

(C) Springer-Verlag Berlin Heidelberg 2011 
attractive as it is invariant to local translation and rotation assuring meaningful regularization through the use of principal stretches; the eigen value of the left and right stretch tensor. The use of this type of regularizer is computational expensive. However, as we show very efficient computations through analytical expressions can be made including their derivatives. The resulting scheme is 1.45 times the computational cost of SSD for tedrahedra in 3D. This enables the use of gradient based methods, with fast convergence. Furthermore, this provides the local scaling (the determinant of the stretch) such that the non-linearity in the transformation can be accounted for in the similarity measure. We present a registration framework that simplifies the implementation of these constraints. The framework is based on the left Cauchy-Green strain tensor and its eigen values (squared principal stretches). We form analytical derivatives of the eigen values with respect to the parameters, independent of the parameterization. This is essential for easy and fast implementation. Through the chain-rule it is possible to easily change bases and elasticity model. We illustrate the method on synthetic data, 2D MRI data and 3D surface registration of Human ear canals. We model the deformation and exemplify some derivations with tetrahedra and B-splines as the bases to illustrate the approach and implementation.

\section{Previous Work}

Registration is an area of continuous research because of its importance in creating a basis for further analysis. The problem is generally ill-posed, so it needs a prior or regularization to obtain meaningful results. In most registration schemes, a regularizer is applied to ensure a valid spatial deformation which is smooth and preserves topology. The majority of regularization approaches find their motivation in continuum mechanics. Linear elastic body forces was initially proposed by 1 to regularize the deformation. This was later adapted into a registration algorithm in [2]. Riemannian elasticity was introduced by [3] which, in contrast to linear elasticity, is rotation-invariant and therefore capable of capturing much larger deformations. Elastic image registration by incorporating volume-preserving soft constraints in registration of pre- and post-contrast MRIs of the female breast was essentially performed in [4] and [5. Similarly, 6] used volume-preserving hard constraints together with linear elasticity to register pre- and post-contrast MRIs. 7] proposed diffusive regularization, which is the squared Fröbenius norm of the displacement gradient. The use of viscous-fluid priors was introduced in [8] which regularize the flow of the deformation rather than the relative spatial displacements. Fluid registration has become widely popular in the neuro-imaging community [8, 9] because of its ability to model large deformations. Rueckert et al. 10] reduced the dimensionality of the image registration problem and ensured a smooth deformation field by using B-splines to describe the deformations between images. Many research groups have since adapted this approach [11,12]. Other typical parameterizations of the deformation field are the cosine kernel proposed by [13] and different kinds of radial basis functions [14. The focus of this work is on generalization of non-linear strain and we exemplify using 
Riemannian elasticity, with a formulation that allows for easy exchange of the regularizer to other forms such as the Ogden material model [15]. The reminder of this paper is organized as follows: In section 3 we briefly review registration, in section 4 we briefly discuss similarity measures and derive the first order structure for SSD as an example. In section 5 we present the regularization framework and present our approach to evaluate the regularization functional and the first order structure. In section 6 we discuss two different commonly used bases, B-splines and tetrahedra. In section 7 we present experimental results, discuss the method in 8 and draw our conclusion in section 9 .

\section{Registration}

We formulate the registration problem as follows: Find a transformation $\phi$ which maps $\boldsymbol{R}$ to $\boldsymbol{I}$ minimizing the similarity measure $\mathcal{D}$. This is an ill-posed problem, so we add a regularization term $\mathcal{S}$ to the function $\phi$. This can be written as the following objective function

$$
\mathcal{F}[I, R, \phi]=\mathcal{D}[R, I \circ \phi]+\alpha \mathcal{S}[\phi],
$$

where $\phi$ is the deformation. Most registration schemes are formulated numerically and the influence of local scaling which occur for non-rigid transformations are left out. Some schemes compensate for this through re-gridding. However when dealing with large deformation as non-linear hyper elasticity a decision about local scaling has to be made, one is to take scaling into account or to consider the problem as re-sampling. For the minimization of $\mathcal{F}$ we use the gradient-based methods, in particular LBFGS [16]

\section{Similarity Measure and Image Function}

The image forces which drive the registration are derived from the similarity between the reference and the deformed template image. The natural choice is sum of squared differences (SSD), however, this requires images where the values are directly comparable without major bias, gradients etc. To counter these effects, several other similarity measures exist. The most important include: mutual information [17, normalized mutual information [18, normalized gradient fields [19], cross correlation [1] and correlation ratio 20. For surfaces, the choice is often SSD on a signed distance field or point-to-point distance (Iterated Closes Point ICP) [21].For first order structure of the similarity measure SSD, we write

$$
\mathcal{D}[I, R, \boldsymbol{\phi}]=\int_{\Omega}(R(\boldsymbol{x})-I \circ \boldsymbol{\phi}(\boldsymbol{x} ; \boldsymbol{p}))^{2} d \boldsymbol{x} .
$$

Differentiating with respect to the parameters $p$ and using the chain rule we get

$$
\frac{\partial \mathcal{D}}{\partial \boldsymbol{p}}=\frac{1}{2} \int_{\Omega}(R(\boldsymbol{x})-I \circ \boldsymbol{\phi}(\boldsymbol{x} ; \boldsymbol{p})) \frac{\partial I(\tilde{\boldsymbol{x}})}{\partial \tilde{\boldsymbol{x}}} \frac{\partial \phi(\boldsymbol{x} ; \boldsymbol{p})}{\partial \boldsymbol{p}} d \boldsymbol{x}
$$

where $\tilde{\boldsymbol{x}}=\boldsymbol{\phi}(\boldsymbol{x} ; \boldsymbol{p})$. Images are considered to be smooth functions ensured by using cubic B-spline interpolation, which is $C_{2}$-continuous. 


\section{Regularization}

We formulate the regularization energy upon $\phi$ as the integration of an energy density function $r$, i.e.

$$
\mathcal{S}[\phi]=\int_{\Omega} r[\phi](\boldsymbol{x}) d \boldsymbol{x},
$$

We prefer the elasticity-based regularizers as they allow for large deformation because of rotation-invariance. Any isotropic elasticity energy density function can be formulated as a function of the eigen values of the left Cauchy-Green strain tensor.

$$
\mathbf{E}(\boldsymbol{x})=\nabla \phi(\boldsymbol{x}) \nabla \boldsymbol{\phi}(\boldsymbol{x})^{T} .
$$

Among these elasticity-based regularizers are volume preservation, Riemanian elasticity and St. Venant Kirchoff elasticity. The density functions are

$$
\begin{aligned}
& r_{v o l}[\phi]=\left(\prod_{i} \epsilon_{i}-1\right)^{2}, \\
& r_{\text {rie }}[\phi]=\frac{\mu}{4} \sum_{i} \log ^{2} \epsilon_{i}+\frac{\lambda}{8}\left(\sum_{i} \log \epsilon_{i}\right)^{2}, \\
& r_{s v k}[\phi]=\frac{\mu}{4} \sum_{i}\left(\epsilon_{i}-1\right)^{2}+\frac{\lambda}{8}\left(\sum_{i}\left(\epsilon_{i}-1\right)\right)^{2}
\end{aligned}
$$

where $\boldsymbol{\epsilon}$ is the eigen values of the left Cauchy-Green strain tensor. The regularizer must be differentiated with respect to the parameters which, as we propose, can be achieved using the chain rule. The purpose is to derive an expression for

$$
\frac{\partial r[\phi]}{\partial p}=\sum_{i} \frac{\partial r[\phi]}{\partial \epsilon_{i}} \frac{\partial \epsilon_{i}}{\partial p}
$$

It is straight forward to compute $\frac{\partial r[\phi]}{\partial \epsilon}$ i.e. for Riemanian elasticity

$$
\frac{\partial r_{\text {rie }}[\phi]}{\partial \epsilon_{i}}=\frac{\mu}{2} \frac{1}{\epsilon_{i}} \log \epsilon_{i}+\frac{\lambda}{4} \frac{1}{\epsilon_{i}} \sum_{i} \log \epsilon_{i}
$$

Thus, our concern is to find a way to compute $\frac{\partial \epsilon_{i}}{\partial p}$, the derivatives of the eigen values w.r.t. the deformation parameters.

\subsection{Eigen Values and Derivatives}

We regularize the strain of the deformation through the eigen values of the strain tensor which can be decomposed as $\mathbf{E}=\mathbf{U} \boldsymbol{\Lambda} \mathbf{U}^{T} . \mathbf{E}=\nabla_{x} \boldsymbol{\phi}(\boldsymbol{x}) \nabla_{x} \boldsymbol{\phi}(\boldsymbol{x})^{T}$ a positive definite symmetric matrix, assuming diffeomorphism. We want to take the derivative with respect to the eigen values $\Lambda$. For eigen values of a symmetric matrix with multiplicity of one it holds

$$
\mathbf{E}=\mathbf{U} \boldsymbol{\Lambda} \mathbf{U}^{t} \Leftrightarrow \frac{\partial \epsilon_{i}}{\partial p}=\mathbf{u}_{i}^{T} \frac{\partial \mathbf{E}}{\partial p} \mathbf{u}_{i}
$$


The change in the strain can be written as

$$
\frac{\partial \mathbf{E}}{\partial p_{j}}=\frac{\partial \nabla_{x} \boldsymbol{\phi}(\boldsymbol{x} ; \boldsymbol{p})}{\partial p_{j}} \nabla_{x} \boldsymbol{\phi}(\boldsymbol{x} ; \boldsymbol{p})^{T}+\nabla_{x} \boldsymbol{\phi}(\boldsymbol{x} ; \boldsymbol{p}) \frac{\partial \nabla_{x} \boldsymbol{\phi}(\boldsymbol{x} ; \boldsymbol{p})^{T}}{\partial p_{j}}
$$

Combining eq. 12 and eq. 11 the change in eigen values can be written as

$$
\frac{\partial \epsilon_{i}}{\partial p_{j}}=\mathbf{u}_{i}^{T}\left(\frac{\partial \nabla_{x} \boldsymbol{\phi}(\boldsymbol{x} ; \boldsymbol{p})}{\partial p_{j}} \nabla_{x} \boldsymbol{\phi}(\boldsymbol{x} ; \boldsymbol{p})^{T}+\nabla_{x} \boldsymbol{\phi}(\boldsymbol{x} ; \boldsymbol{p}) \frac{\partial \nabla_{x} \boldsymbol{\phi}(\boldsymbol{x} ; \boldsymbol{p})^{T}}{\partial p_{j}}\right) \mathbf{u}_{i}
$$

For multiplicity greater than one, no unique eigen vector exist however the derivatives are easily dealt with as follows. As we assume diffeomorphism the multiplicity simply indicates isotropy on a hyper plane. Thus, orthogonal to the distinct eigen vectors we find a hyper plane on which the stretch is the same in any direction. We can therefore freely choose the vectors in this hyper plane since all will be eigen vectors, thus the directions of the derivatives are free within this plane due to isotropy. These special cases can be dealt with easily in the implementation. Alternatively explicit derivatives exist for the SVD [22].

\section{Choice of Basis Functions}

The choice of function for $\phi$ has a huge impact on performance, so both interpolating and non-interpolating functions can be used. We formulate the problem for a general basis function for $\phi$ which models the local neighborhood transformation and is evaluated at a single point $\boldsymbol{x}$. The local transformation of $\boldsymbol{x}$ can be written as follows.

$$
\phi(\boldsymbol{x} ; \boldsymbol{p})=\boldsymbol{x}+\boldsymbol{B}(\boldsymbol{x}) \boldsymbol{p}
$$

where $\mathbf{p}$ is the parameters of $\boldsymbol{\phi}(\boldsymbol{x}, \boldsymbol{p}) . \boldsymbol{\phi}$ characterizes the local deformation at $\boldsymbol{x}$. The Jacobian $\boldsymbol{\nabla} \boldsymbol{\phi}=\boldsymbol{J}$ can then be written as

$$
\begin{aligned}
\nabla_{x} \phi(\boldsymbol{x} ; \boldsymbol{p})= & \boldsymbol{I}+ \\
& {\left[\begin{array}{lll}
\boldsymbol{B}_{1}(\boldsymbol{x}) \boldsymbol{p} & \ldots & \boldsymbol{B}_{N}(\boldsymbol{x}) \boldsymbol{p}
\end{array}\right], } \\
\nabla_{p} \boldsymbol{\phi}(\boldsymbol{x} ; \boldsymbol{p})= & \boldsymbol{B}(\boldsymbol{x}),
\end{aligned}
$$

where $\boldsymbol{B}_{i}(\boldsymbol{x})=\frac{\partial \boldsymbol{B}(\boldsymbol{x})}{\partial x_{i}}$. Several choices of basis functions exist and the above formulation does not limit the choice. For bases such as tensor product B-splines as used in 10, cosine basis as used in 23] or polyhedra it is fast and straight forward.

\subsection{Basis Examples}

As examples we formulate the problem with tetrahedra and B-splines as the basis function which models the local neighborhood. Tetrahedra models an affine transformation, and we represent this by a single point $\boldsymbol{x}$, the barycenter [24] of the tetrahedra for deformation constraint, and the points as the parameters 

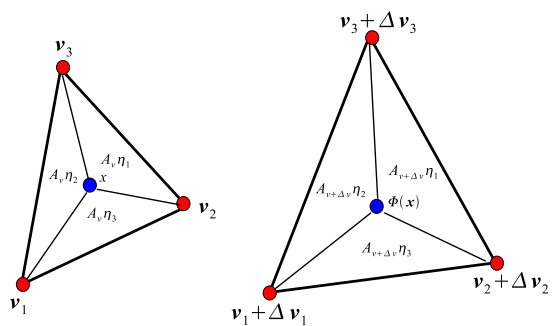

Fig. 1. Illustration of the linear/affine transformation from triangle $\mathbf{v}_{\mathbf{1}}, \mathbf{v}_{\mathbf{2}}, \mathbf{v}_{\mathbf{3}}$ to $\mathbf{v}_{1}+\Delta \mathbf{v}_{1}, \mathbf{v}_{2}+\Delta \mathbf{v}_{2}, \mathbf{v}_{3}+\Delta \mathbf{v}_{3}$

in the transformation i.e. a free form deformation for all sample points with a constraint formulated for the barycenter. Figure 1 shows the a deformation based on triangles in $\mathbf{x}$ can be written as a linear combination of the vertices $\boldsymbol{V}$ in the tetrahedra $\boldsymbol{x}=\left[\boldsymbol{v}_{\mathbf{1}}, \ldots, \mathbf{1}_{\boldsymbol{n}}\right] \boldsymbol{\eta}=\boldsymbol{V} \boldsymbol{\eta}$ thus $\boldsymbol{\eta}=\boldsymbol{V}^{-1}\left[\begin{array}{c}\boldsymbol{x} \\ 1\end{array}\right]=\left[\boldsymbol{D} \boldsymbol{\eta}^{0}\right]\left[\begin{array}{c}\boldsymbol{x} \\ 1\end{array}\right]$ where $\boldsymbol{\eta}$ is the barycentric coordinates of $\boldsymbol{x}$. Thus by choosing polyhedra as the basis, the analytical derivations of the objective function and its first order derivative becomes feasible. By first splitting the position of $\boldsymbol{x}$ up we write $\boldsymbol{x}=\boldsymbol{x}_{0}+\Delta \boldsymbol{x}$, the deformation and the Jacobian $\boldsymbol{\nabla} \phi=\boldsymbol{J}$ can then be written as

$$
\phi(x)=\mathrm{x}+\Delta \mathbf{P} \eta=x+\Delta \boldsymbol{P}\left[\boldsymbol{D} \eta_{0}\right]\left[\begin{array}{l}
\boldsymbol{x} \\
1
\end{array}\right] \quad \nabla_{x} \phi(x)=\boldsymbol{I}+\Delta \boldsymbol{P D}
$$

Similarly for B-splines the derivatives can be derived. As B-splines are tensor product basis function we restrict ourselves to the 1-dimensional case. The basis polynomials and derivatives are given by:

$$
\boldsymbol{B}(t)=\left\{\begin{array}{c}
-t^{3}+3 t^{2}-3 t+1 \\
3 t^{3}-6 t^{2}+4 \\
-3 t^{3}+3 t^{2}+3 t+1 \\
t^{3}
\end{array}\right\}, \Delta \boldsymbol{B}(t)=\left\{\begin{array}{c}
-3 t^{2}+6 t-3 \\
9 t^{2}-12 t \\
-9 t^{2}+6 t+3 \\
3 t^{2}
\end{array}\right\}, 0 \leq t<1(
$$

thus we write $\phi(t)=t_{0}+\boldsymbol{B}(t) \boldsymbol{p}$ where p are the parameters and $\nabla_{t} \phi=1+$ $\Delta \boldsymbol{B}(t) \boldsymbol{p}$. The extension to $N D$ is straight forward.

\section{$7 \quad$ Experiments}

Two types of experiments have been conducted. One type is on synthetic data to illustrate the ability to handle large deformations. We use $2 \mathrm{D}$ registration with B-splines and a 3D simulation using tetrahedra. For the real data we apply correlation ratio (CR) for MRI as distance measures and the SSD of a signed distance map for the ears. For all real data experiments a scale space approach has been used in a coarse to fine manner for the deformation, thus propagating the deformation field to a set of basis functions with higher resolution. 


\subsection{Synthetic Data}

Several experiments on synthetic data are presented to illustrate the properties of the elasticity in this formulation for large-scale deformation. 2D experiments with 3rd degree B-splines and 3D using tetrahedra.

Large deformations. To test the regularization and its ability to capture largescale deformation we have performed three experiments. We register a square to a disc, a $\mathrm{C}$ to a disc and a disc to a $\mathrm{C}$ (figure 2). The experiments are performed in $2 \mathrm{D}$ with a uniform B-spline basis as parameterization of the deformation $\phi$ and cubic B-spline image interpolation.For the first experiment we use a resolution of 10 pixels between each node in the B-spline on the images with resolution $300 \times 300$ pixels. The number of samples are uniformly $100 \times 100$ in each direction. As figure 2(a) show, we can register a square to a circle obtaining smooth deformation fields that is diffeomorphic by definition. Registration of a $\mathrm{C}$ to a disc and vice versa is a difficult task and the success heavily depends on the right choice of scale for the basis functions. In this experiment we use 60 pixels between nodes $30,8,4$, and 2 to obtain the desired result without falling into a local minimum. As figure 3(b) and figure 3(c) shows, we obtain deformation fields for both $\mathrm{C}$ to disc and disc to $\mathrm{C}$ with very large deformations. The obtained deformation fields are guaranteed to be diffeomorphic. To obtain symmetric solution such as in figure $3(\mathrm{a})$ all of the basis functions at each resolution must be placed exactly symmetric and the problem must be exactly symmetric. Otherwise the solution will be slightly asymmetric, emphasizing slightly.

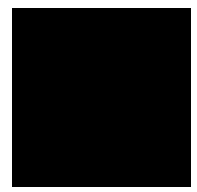

(a) Square

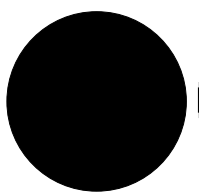

(b) Disk

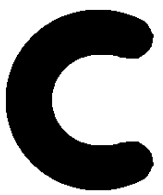

(c) $\mathrm{C}$

Fig. 2. The square (a) and the $\mathrm{C}(\mathrm{c})$ have been registered to the disc (b)

Tetrahedra. To show the efficiency of the model and its ability to handle large deformations we have deformed a $3 \mathrm{~d}$ cube of 6000 tetrahedra under constant force. The result in figure 4(b) is obtained without numerical instability and handles as can be seen very large deformations. Over 80 evaluations we compared the hyper elastic energy and SSD which gave a ratio of computational time of $\mathrm{HE} / \mathrm{SSD}=1.45$ which shows the efficiency of the algorithm.

\subsection{Cardiac Data}

This data is a part of a cardiac data set of patients with a serious heart condition. The data consists of $2 \mathrm{D}$ slices from different patients which we co-register using $\mathrm{CR}$ and B-spline parameterization of $\phi$. The slices are not obtained in exactly 


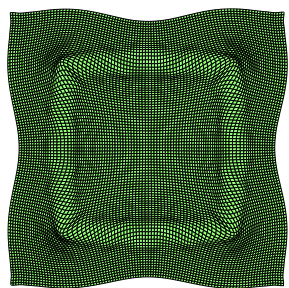

(a) Square to disc

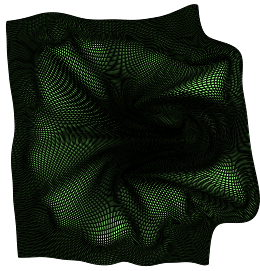

(b) C to disc

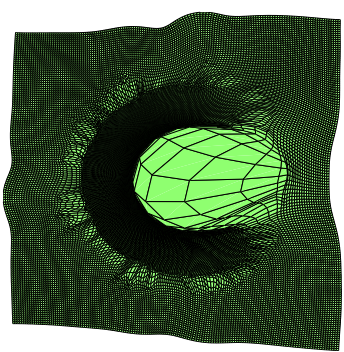

(c) Disc to C

Fig. 3. (a)The deformation field for the registration of the square registered using Riemanian elasticity and B-spline.(b) The deformation field of the $\mathrm{C}$ registered to the disc using Riemanian elasticity and B-spline. (c) The deformation field of the disc registered to the $\mathrm{C}$ using Riemanian elasticity and B-spline.

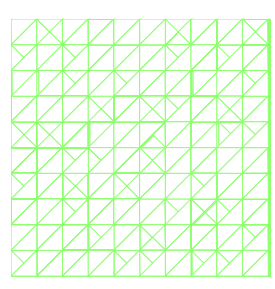

(a)

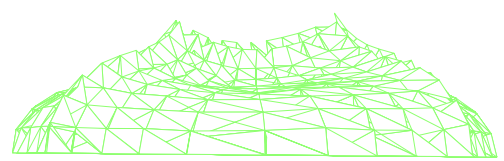

(b)

Fig. 4. (a)The un-deformed cube (b) the deformed cube. The transformation to $\mathbf{b}$ from $\mathbf{a}$ is guaranteed to be diffeomorphic.

the same angle relative to the patient. Therefore most of the background in the images has been removed for demonstration purposes. The classes for the CR is based on a reference image segmented by hand. Figure [5] show a registration results, the transformation, the residual and the segmentation difference. The results show that the elasticity forces help move the papillary muscles into place as we know that they are considered a part of the myocardium by the CR due to the segmentation.

\subsection{Ear Data}

In addition we register $803 \mathrm{D}$ surfaces representing ear impression using 3D hyper elastic prior. This data was presented in 25] and was obtained to analyze the shape changes induced in the ear canal by movement of the mandible. The resulting average shape and a random deformation field is shown in figure 6 . 


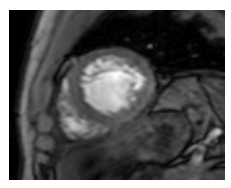

(a) Reference

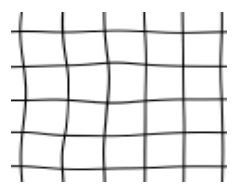

(b) Transformation

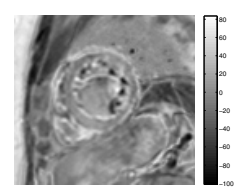

(c) Residual

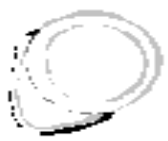

(d) Dice image

Fig. 5. (a) The reference image. (b) The transformation. (c) The intensity residual showing that the papillary muscles are registered correctly. (d) The difference in segmentations after registration.

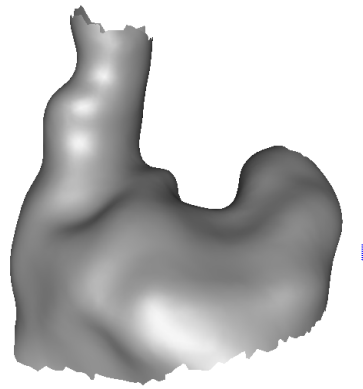

(a) Average ear

(b) Deformation field

Fig. 6. (a) The average ear from 80 ears. (b) A $3 \mathrm{D}$ deformation field from registration of 2 ears.

\section{Discussion}

The method described in this paper can be implemented in parallel and offers due to the chain rule an easy way of switching between different kinds of elasticity. The performance in highly dependent on the parameterization $\phi$ e.g. a B-spline in 3D will have 192 parameters where as the tetrahedra only has 12 from which the strain tensor is computed. Thus, one should when solving a specific problem carefully select the appropriate parameterization such that speed, accuracy and performance suit the given task.

\section{Summary and Conclusion}

We have presented an efficient way of implementing hyper elastic regularization based on the chain rule and the derivatives of the eigen values of the left CauchyGreen strain tensor. The method is illustrated on Riemanian Elasticity but it is in no way limited to this. We have shown in practice how the methodology 
is adapted to different parameterizations of the deformation and the fact that the approach can generally be adapted to any parametric base. Finally we have successfully applied the method to both synthetic and real data, in so doing illustrating the properties of the regularization. These include the ability to capture large deformation such as registering a $\mathrm{C}$ to a disc. The regularization can also capture more subtle deformation such as the deformation between ears in a population and it can capture very small local deformations such as the MRI data. Finally we have illustrated the effectiveness of the approach by comparing it to the computational complexity of SSD. The results were very convincing and only 1.45 times as computational expensive as SSD using B-spline interpolation.

\section{References}

1. Broit, C.: Optimal Registration of Deformed Images. PhD thesis, University of Pennsylvania (1981)

2. Bajscy, R., Lieberson, R., Reivich, M.: A computerized system for the elastic matching of deformed radiographic images to idealized atlas images. Computer assisted Tomography 5, 618-625 (1983)

3. Pennec, X., Stefanescu, R., Arsigny, V., Fillard, P., Ayache, N.: Riemannian Elasticity: A Statistical Regularization Framework for Non-linear Registration. In: Duncan, J.S., Gerig, G. (eds.) MICCAI 2005. LNCS, vol. 3750, p. 943. Springer, Heidelberg (2005)

4. Rohlfing, T., Maurer Jr., C., Bluemke, D., Jacobs, M.: Volume-preserving nonrigid registration of MR breast images using free-form deformation with an incompressibility constraint. IEEE Transactions on Medical Imaging 22(6), 730-741 (2003)

5. Tanner, C., Schnabel, J., Degenhard, A., Castellano-Smith, A., Hayes, C., Leach, M., Hose, D., Hill, D., Hawkes, D.: Validation of Volume-Preserving Non-rigid Registration: Application to Contrast-Enhanced MR-Mammography. In: Dohi, T., Kikinis, R. (eds.) MICCAI 2002. LNCS, vol. 2488, pp. 307-314. Springer, Heidelberg (2002)

6. Haber, E., Modersitzki, J.: Numerical methods for volume preserving image registration. Inverse Problems 20(5), 1621-1638 (2004)

7. Fischer, B., Modersitzki, J.: Fast diffusion registration. AMS Contemporary Mathematics, Inverse Problems, Image Analysis, and Medical Imaging 313, 117-129 (2002)

8. Christensen, G.: Deformable shape models for anatomy. Electrical Engineering D. Sc. Dissertation, Washington University, St. Louis, Missouri (1994)

9. Cardenas, V., Studholme, C., Gazdzinski, S., Durazzo, T.C., Meyerhoff, D.J.: Deformation-based morphometry of brain changes in alcohol dependence and abstinence. NeuroImage 34, 879-887 (2007)

10. Rueckert, D., Sonoda, L.I., Hayes, C., Hill, D.L.G., Leach, M.O., Hawkes, D.J.: Nonrigid registration using free-form deformations: application to breast MR images. IEEE Transactions on Medical Imaging 18(8), 712-721 (1999)

11. Studholme, C., Constable, R., Duncan, J.: Accurate alignment of functional EPI data to anatomical MRI using aphysics-based distortion model. IEEE Transactions on Medical Imaging 19(11), 1115-1127 (2000)

12. Vester-Christensen, M., Erbou, S.G., Darkner, S., Larsen, R.: Accelerated 3D image registration. In: SPIE Medical Imaging 2007 (2007) 
13. Cootes, T., Twinning, C., Taylor, C.: Diffeomorphic Statistical Shape Models. In: British Machine Vision Conference, vol. 1, pp. 447-456 (2004)

14. Bookstein, F.L.: Principal warps: thin-plate splines and the decomposition of deformations. IEEE Transactions on Pattern Analysis and Machine Intelligence 11(6), 567-585 (1989)

15. Ogden, R.W.: Large Deformation Isotropic Elasticity-On the Correlation of Theory and Experiment for Incompressible Rubberlike Solids. Proceedings of the Royal Society of London. Series A, Mathematical and Physical Sciences (19341990) 326(1567), 565-584 (1972)

16. Liu, D.C., Nocedal, J.: On the limited memory BFGS method for large scale optimization. Mathematical programming 45(1), 503-528 (1989)

17. Wells, W., Viola, P., Atsumi, H., Nakajima, S., Kikinis, R.: Multi-modal volume registration by maximization of mutual information. Medical Image Analysis 1(1), 35-51 (1996)

18. Studholme, C., Hill, D., Hawkes, D.: An overlap invariant entropy measure of 3D medical image alignment. Pattern Recognition 32(1), 71-86 (1999)

19. Haber, E., Modersitzki, J.: Intensity Gradient Based Registration and Fusion of Multi-modal Images. Methods of Information in Medicine 46(3), 292 (2007)

20. Roche, A., Malandain, G., Pennec, X., Ayache, N.: The Correlation Ratio as a New Similarity Measure for Multimodal Image Registration. In: Wells, W.M., Colchester, A.C.F., Delp, S.L. (eds.) MICCAI 1998. LNCS, vol. 1496, pp. 1115-1124. Springer, Heidelberg (1998)

21. Besl, P., McKay, N.: A method for registration of 3-D shapes. IEEE Transactions on Pattern Analysis and Machine Intelligence 14(2), 239-256 (1992)

22. Papadopoulo, T., Lourakis, M.: Estimating the Jacobian of the Singular Value Decomposition: Theory and Applications. In: Vernon, D. (ed.) ECCV 2000. LNCS, vol. 1842, pp. 554-570. Springer, Heidelberg (2000)

23. Cootes, T., Marsland, S., Twining, C., Smith, K., Taylor, C.: Groupwise diffeomorphic non-rigid registration for automatic model building. In: Pajdla, T., Matas, J(G.) (eds.) ECCV 2004. LNCS, vol. 3024, pp. 316-327. Springer, Heidelberg (2004)

24. Möbius, A.F.: Der barycentrische Calcul: ein neues Hülfsmittel zur analytischen Behandlung der Geometrie: mit 4 Kupfertafeln, JA Barth (1827)

25. Darkner, S., Larsen, R., Paulsen, R.: Analysis of deformation of the human ear and canal caused by mandibular movement. In: Proceedings of the 10th International Conference on Medical Image Computing and Computer-Assisted Intervention, pp. 801-808. Springer, Heidelberg (2007) 